\title{
Membership Directory
}

NALTD

1972 MEMBERSHIP DIRECTORY

(Corrected to February 1, 1972)

Permission for Commercial Use Must Be Obtained from the NALED Executive Secretary

PAST PRESIDENTS

Thomas R. Goldsworthy

Iouis Chatagnier

Evelyn Uhrhan Irving

ABALLI, Dolores, DLI, St Mary's College, Notre Dame, In 46556 ABOYAN, H J, 610 Cedar Grove Rd, Broomall, Pa 19008

ADAM, Richard, DLL, Western Reserve Academy, Hudson, Oh 44236

ADAMS, Kenneth R, Weber State College, FL Dept, Ogden, Ut 84403

ADAMS, Mrs Sudie $M$, Asst Prof Span and Port, Baylor Univ, Box 159, Waco, Tx 76703

ADAMSON, Charles F, 821 Osceola Ave, St Paul, Mn 55105

AGATSTEDN, Michael, 1700 st Marks Ave, Merrick, N Y 11566

AGOSTINo, Nellie, Chmn FL Dept, East Hart High, 777 Burnside Ave, E Hartford, Ct 06117

AGUIRRE, A A, Span Dept Head, I U at South Bend, Northside Blvd, South Bend, In 46615

AIREN, John A, Westchester Comm College, 75 Grasslands Rd, Valhalla, NY 10595 AIKENS, H F, DLL, Dalhonsie Univ, Halifax, Nova Scotia, Canada

ALAMEDDINE, Farouk B, DLL, Wayne State Univ, 20023 Trinity Ave, Detroit, Mi 48219

ALCORN, Kent, 7723 Chambers Creek Rd, Tacoma, Vash 98467

ALTMAN, Howard B, Dept Ger Lang, Univ of Washington, Seattle, Wa 98105

ALTMAN, Lee (Leon), DFL, San Frencisco State College, 1600 Holloway, San Francisco, Ca 94132

AMBERT, Gary John, DLL, E Carolina Univ, Greenville, N C 27834

AMOR, Sr Rose Teresa, Chmn Span Dept, Molloy Catholic College, Rockville CTP, NY 11570

ANNABEL, Patricia C, Chmn FL Dept, Slippery Rock State College, Slippery Rock, $\mathrm{Pa} 16057$

APPLEBAUM, Lee, Seattle Comm College, 401 queen Anne Ave N, Seattle, Wa 98109

ARBAIZA, N D, Chmn, Dept FL, Rockland Comm College, 145 College Rd, Suffern, NY 10901

ARENDT, J, Cnslr in FL, Minneapolis Schools, 3709 48th Ave S, Minneapolis, Non 55406

ARENSMAN, Imogene C, Chmn FL, Metropolis Comm HS, 1004 Catherine St, Metropolis, Il 62960

ARMISTEAD, Elizabeth, Iees-McRae College, Box 618, Banner Elk, NC

ASH, F C, DLL, 3904 Vardell Rd NW, Calgary, Alberta, Canada

ASSELIN, GuY, DLL, Carleton Univ, Ottawa 1, Ontario, Canada

ASSELT, J an Van, head FL Dept, McPherson College, McPherson, Ks 67460

ATKTISON, Mrs Geo M, 1529 Denver Ave, Apt 10, Kansas City, Mo 64127

ATTIG, Cecil E, Supv, California State College, $800 \mathrm{~N}$ State College Blvd, Fullerton, Ca 96231

AUCOIN, G E, 1.929 Brimfield Rd, Columbus, On 43229

AUGER, Gilles E, Nasson College, Springvale, Me 04033 


\section{Membership Directory}

AUSTIN, Robert P, DLL, Loyola Academy, $1100 \mathrm{~N}$. Laramie, Wilmette, Il 60091 AYALA, Mrs. A, Span Prof, Rollins College, Box 119, Winter Park, Fl 32739

$$
--\mathrm{B}--
$$

BAAK, Dr L E, Chmn Dept Mod Lang, Morningside College, Sioux City, Ia 51106 BABIN, J W, Assoc Prof of French, St F X Univ, Antigonish, Nova Scotia, Canada BAECHIE, Mary, FL Chmn, C L Comm HS, $45 \mathrm{~W}$ Franklin St, Crystal Lake, Il 60014 BAIMAS, John C, Lang Dept Head, Fitchburg HS, Fitchburg, Ma 01420

BAKER, Reid E, Ohio Dept of Education, 781 Northwest Blvd, Columbus, oh 43212 BAKER, Robert I, Chmn Dept of Russian, Middlebury College, Middlebury, vt 05753

BALBI, Mario, Benedictine Mili school, 6502 Seawright Dr, Savannah, Ga 31406 BAICAEN, Hubert, Lect, Univ of Manitoba, 500 Dysart Rd, Winnepeg 19, Manitoba, Canada

BALL, Mrs Jeannie, Chmn, French Dept, Madeina School, Greenway, Va 22067 BALIINS, Ms Vivija N, P O Box 1012, Ft Wayne, In 46801

BALTIS, Paul P, LL Supv, Ieeward Comm College, Pearl City, Hi 96782

BANER, Hugo, Lindenwood College, St Charles, Mo 63301

BARGER, Kenneth S, DLL, Columbia Corm Unit 4, P O Box 1, Columbia, Il 62236

BARGER, Sharon, DLL, Woodstock HS, Woodstock, II 60098

BARICEVIC, Elizabeth M, Maryhurst College, Maryhurst, or 97036

BARR, Charles J, DIL, St John S College, 5118 Seminary Rd, Cemarillo, Ca 93010

BARRIE, Pedre, DLI, Macon Jr College, Macon, Ga 31206

BARRUTIA, Dr Richard, Univ de California, Apartado Postal 6-394, Mexico 6 D F, Mexico

BARTH, Rev Gilbert, TOR, Head, Dept of Mod Lang, College of Steubenville, Steubenville, on 43952

BASSETT, Relph W, Supv FL, Portland Public Schools, $631 \mathrm{NE}$ Clackamas St, Portland, or 97208

BAVER, Dr Eric W, Lang Serv, Monash Univ, Clayton, Victoria, Australia 3168

BEAUDOIN, Henri, AV Dir, Cegep de Rouyn-Noranda, P 0 Box 1500, Rouyn P, Quebec, Canada

BECK, Mrs Francis, Univ of Chicago, 5832 S Stoney Island, Chicago, Il 60637 BEECHER, Mrs Jean, Moravian College, Bethlehem, Pa 18018

BEIER, Diane, 709 Timpe Rd, Fremont, Ohio 43420

BELL, Keith R, Prof Mod Lang, Mid-American Nazarene College, 2030 College Vay, Olathe, Ks 66061

BERERSDORFER, William, Ohio State Univ, Listening Center, Columbus, oh 43210

BERNINE, Sr M, FSPA-APOF, Viterbo College, 815 s 9th St, La Crosse, Wi 54601

BERTEAU, Mrs Doris, Horlick HS, 2119 Rapids Dr, Racine Wi 53404

BERTRAND, Frere Alphonse, La Bibliotheque, Ecole Normale HS de Foy, Cap-Rouge, Quebec, Canada

BISHOFF, Elizabeth M, C G D, Randolph-Macon Woman's College, R-M W C, Lynchburg, Va 24503

BLAIR, ioseph C, DLL, Lang Lab A-S, R 3, Univ of Missouri, Columbia, Mo 65201 BLATCHFORD, Charles H, 476 Kekupua St, Honolulu, Hi 96821

BODLANDER, Hans, LU Instr, U of Bridgeport, Dana Hall, Bridgeport, Ct 06602 BOLDUC, Prof R, Head, Lang Dept, 251 Belmont St, Quinsigamond Comm College, Worcester, Ma 01605

B00T, Christine, Asst Prof, 6905 18th Ave, Kenosha, Wi 53140

BOOTHE, Robert O, Dir FL, California State Polytechnic College, San Luis Obispo, Ca 93401

BORN, Warren C, Asst Dir MLA/ERIC, 62 Fifth Ave, New York, NY 10011

BORRA, Supv FL, Warwick Vet Mem HS, Warwick, RI, 02896

BOSTIAU, Herbert, Box 8198 UND Station, Grand Forks, ND 58201

BOUCHER, John G, DLL, St Anselm S College, Hanchester, NH 03102

BOUDREAU, Mrs Jacqueline, Supv LL, Wheaton College, Norton, Ha 02766

BNUSARD, Roger, DLL, North Carolina College, 1406 i/2 Clarendon, Durham, NC 27705 


\section{Membership Directory}

Boulos, H Chas, Dir AV Dept, St Jean-Sur-Richelieu, Box 310, St Jean, PR, Canada

BOURAOUI, $\mathrm{Dr} H \mathrm{~A}$, French lit Dept, 232 Founders College York U70, 4700 Keele St, Downsview, Ontario, Canada

BOWEN, Louise H, DLI, Dartmouth College, 201 Bartlett Hall, Hanover, NH 03755

BOYDEN, Patrick C, DLI, Kent State Univ, Kent, Ohio 44240

BRADLEY, Mrs Paula, Chmn Dept FL, Principia College, Elsah, Il 62028

BRATNOBER, H L Jr, Chmn FL Program, SW Minnesota State College, Marshall, Mn 56253

BRETT, Kenneth C, Dept FL, Univ of Montana, Missoula, Mt 59801

BRIGGS, Mrs Becky A, P O Box 65619, Michigan State Univ, LL A-126 Wells Hall, E Lensing, Mi 43923

BRITTAIN, Mrs F C, W Chicago Comm HS, 326 Joliet St, W Chicago, Il 60185

BROSSMAN, Dr Luther F, Albright College, Reading, Pa 19604

BROWN, C P, FL Dept, Western Kenturky Univ, Bowling Green, Ky 42101

BRONN, Mrs Florence G, DLI SW Biggs Field, Fort Bliss, Tx 79918

BRONN, R L, Asst Vice Dir, Uniao Cul Brasil EU, Fundaçao Brasileira, Rua Col, Oscar Porto 203, Sao Paulo, S P Brasil

BROWN, Robert, FLL Dir, DC Teachers College, Div FL, llth and Harvard Sts NW, Washington, DC 20009

BRUNER, Wilbur J, William Jewell College, Liberty, Mo 64068

BRUST, Mrs J une P, UM Sentor HS, Terwood Rd, Willow Grove, Pa

BRYDON, Mrs M A, Humanities Div, Mount Royal College, Calgary, Alberta, Canada BSHARAH, Sr M Alban, DLL, College of New Rochelle, New Rochelle, N Y 10801

BUCK, Dr George C, DLL, Univ of Washington, 118 Denny Hall, Seattle, Wa 98105 BURR, Yolanda, RD No 3, Hellsville, NY 14895

BUSHALLOW, Thomas, Sup FL, Shaker HS, Latham, NY 12110

BYLINSKI, E F, Institute de ling APP, 25 Rue du Soleil, 67-Strasbourg, France BYRNE, Prs William III, Franklin and Marshall College, Box 151, Lancaster, $\mathrm{Pa} 17603$

$$
--\mathrm{c}--
$$

CALLANAN, James C, DLL, Univ of New Hampshire, Murkland Hall, Durham, NH 03824

CAMPBELL, Mrs G M, Senjor HS, 160 Broad St, Bloomfield, ND 07003

CANDIOTTI, Joseph, United Nations Dev Prog, P O Box 24, Magadiscio, Somalia, East Africa

CANNADAY, Robert V, Jr, FL Section, P O Box 2360, Honolulu, Hi 96804

CAPRETZ, Pierre J, DLL, Yale Univ, 111 Grove St, New Haven, ct 06510

CARMICHAEL Max, Dir of Audio Services, $607 \mathrm{v} 77 \mathrm{th}$ St, Indianapolis, In 46260

CARRIER, Joseph A, 34 Birch St, Biddeford, Me 04005

CARTIER, F A, 1029 Forest Ave, Pacific Grove, Ca 93950

CARTER, Kenneth, Long Beach City College, 701 Iocust Ave, Long Beach, Ca 90913

CERVIZZI, Christine E, C F L, Northbridge HS, Linwood Ave, Whitinsville, Ma 01588

CHANG, Gerald K J, 4457 Puu Panini Ave, Honolulu, Hi 96816

CHATAGNIER, Louis J, 4974 Cote Des Neiges, Apt 1401, Montreal PQ, Canada

CHEATHAM, Rosalie M, 6405 Evergreen Rd, Iittle Rock, Ar 72207

CHERRY, Charles Maurice, Apt 416 Englebart Hall, 1915 Maple Ave, Evanston, Il 60201

CHIIDS, Lora E, DLL, Wilson HS, 1151 SW Vermont St, Portland, or 97219

CHOMEI, Toshiko, Instr, Ochanomizu Univ, Otsuka 2 chome, Bunkyo-Ku, Tokyo, Japan

CHRISTMAN, Henry R, DLI, Kutztown State College, 215 wellington Ave, Reading, $\mathrm{Pa} 19609$

CICERo, A E, Prof, Allegheny Conm College, 308 Ridge Ave, Pittsburgh, Pa 15212

CIRUTI, Miss Joan E, Chmn, Dept of Span, Mt Holyoke College, S Hadley, Ma 01075

CLAESGES, Dr A $\mathrm{w}$, Dept of FL and Lit, S Methodist Univ, Dallas, Tx 75222 


\section{Membership Directory}

CLAPER, Hilliam, MFL Cnslt, Mo State Dept of Ed, Jefferson City, Mo 65101 CLARK, Ben T, Coord Lang Studies, Univ of California, Merrill CollegeuCSC, Santa Cruz, Ca 95060

CLARK, Edward P, Asst Prof, Moorhead State College, Moorhead, Mn 56560

CLARK, Richard A, Butte Junior College, P O Box 566, Durham, Ca 95938

CLINE, TheIma, DLL, Harvey Hall Basement, Adelphi Univ, Garden City, NY Il530

COBB, Mrs J V, 209 Black Bluff Ra, Rome, Ga 30161

COKER, Laura G, $909 \mathrm{~N} 26$ th St, Las Vegas, Nv 39101

COLECCHIA, Dr Francesca, DLL, Duquesne Univ, Pittsburg, Pa 15219

COLL, Pauline, Chmn FL, Averett College, 135 Mulberry Rd, Danville, va 24541

CoLfIrs, Ralph S, Prof, Chrm, Dept FL, Maryville College, 1741 Linda Lane, Maryville, Tn $j 7801$

COLOMBO, Ruth W, Lang Dept, Natick HS, $15 \mathrm{w}$ St, Natick, Ma 01760

CONNORS, Paul R, FL Coord, Randolph High, $194 \mathrm{H} \mathrm{St,} \mathrm{S} \mathrm{Boston,} \mathrm{Ma} \mathrm{021.27}$

CONSULT, Mrs E Alonso, FL, 1410 NE 2nd Ave, Rm 300, Miami, FI 33132

CONGELL, Dr Marilyn J, DLL, Rosemont College, $251 \mathrm{k}$ Dekalb Pike, King of Prussia, Pa 19406

COON, Rev David P, Adm, Iolani School, 563 Kamoku St, Honolulu, Hi 96814

COOPER, C T, Asst Prof of Span, Iebanon Valley College, Annville, Pa 17003

COOFER, Henry F, DLI, Univ of Oregon, Rom Lang Dept, Eugene, Or 97403

CORONADO, Roland, $4023 \mathrm{~N}$ Oriole Ave, Norridge, II 60634

CORPACI, John, Chmn, Lang Dept, Cheshire Acad, Box 306, Cheshire, Ct 06410

CORRY, Bro H Michael, Fsc, DLL, Christian Bro Academy, Lincroft, N J 07738

CORTES, Eladio, DIL and Chmn Span Dept, Rutgers Univ, Camden, N J 03102

COSTA, Ronald K, DLL, Mass St College, Framingham, Ma 01701

COTE, Norman $R$, North Shore Comm College, 3 Essex St, Beverly, Ma 01915

COURNOYER, Madeleine A, Chm FL, Provios East FS, First Ave and Madison, Maywood, Il 60153

COURCIX, M Gerard, College Marie-Victorin, Montreal 462, quebec, Canada

COVE, J I, Dept of French, Queen UnIV, Kingston, Ontario, Canada

COX, Mrs Shirley O, DLL, 8030-B Haley Center, Dept of FL, Auburn Univ 36830

CRAIGHILL, Kary Mute, 4533 38th St S, St Petersburg, FI 33711

CREAMER, John F, DLI, hudson HS, Cottage St, Hudson, Ma 01749

CREAN, John E Jr, Assoc Prof, Dept Eur Lang and Lit, $U$ of Hawaii, Moore Hall, 1890 East-West Rd, Honolulu, Hi 96822

CREE, Raymond Jr, Iibrary, 1011 Vista Chino, Palm Springs, Ca 92662

CRDMINALE, Ieonard R, DLE, Elmira College, Elmira, NY 14901

CRONI, John, Land Coor, Maine Endwell CS, Endwell, NY 13760

CROWNER, Dr David, DLL, Gettysburg College, Gettysburg, Pa 17325

CRUVELLIER, J P A, Head, Mod Lang Dept, Lower Canada College, 4090 Royal Ave, Montreal 261, Canada

CUNEO, Williom J. DLC, Holyoke Corm College, 170 Sargeant St, Holyoke, Ma 01040

CUNNINGHAM, M J Jr, Chrn, Lang Dept, New Mexico Jr College, Lovington HS, Hobbs, IM 88240

$$
--D--
$$

DAIIEY, Bro Edw, Dept Chmn, St Edward HS, 13500 Detroit Ave, Lakewood, On 44107

d'AMCNILLE, Emanuel, Chmn FL Dept, Thayer Academy, 745 Washington st, Braintree, $\mathrm{Ma} 02185$

DALY, Mrs Ruth V, 700 N Main, Ada, Oh 45810

DARRAS, Michael, Transl Bur, Centennial Bldg, Fredericton, NB, Canada

DAVISON, Walter F, Old Dental Clinic, Univ of Pittsburgh, Pittsburgh, Pa

DAY, Philip Stephen, Asst Prof, French, Queen S Univ, Kingston, Ontario, Canada

de BONE, Dr G, Head, Dept FL, La Grange College, La Grange, Ga 30240

DECATOLI, Miss Dolores, Lasalle-Peru Twp HS, 541 Chartres St, LaSalle, II 61301 
DELANOEYE, Mrs john H, FLU, California State College, 5151 state College Dr, Ios Angeles, Ca 90032

DEMAINE, Mr B, 6971. Monkland, Apt 8, Montreal 262, Canada

de PEAUX, Rev R C, Chmn, Dept of MFL, st Norbert College, DePere, wi 54170

de SALVO, Bennie T, Hall High, $50 \mathrm{~s}$ Main St, W Hartford, Ct $0605 i$

DESSUREAUALT, N F, Montreal Catholic School Comm, Adult Ed, 3737 E Sherbrooke St, Montreal 406, Quebec, Canada

DETI, Dr Daniel, Chmn Lang Dept, College of the Desert, Palm Desert, Ca 92260 de TORRE, Emilio E, Queens College of the City of N Y, Flushing, N Y 11367 DEURLOO, Mrs iN C, Univ of Alberta, Rom II, Edmonton, Alberta, Canada DEVINE, Rev F J, S J, Chmn Dept French, St Mary S Univ, Halifax N S, Canada DICICCIO, Miss Elaine, Chmn FU Dept, Concord-Carlisle HS, Concord, Ma 01742 DICKERMAN, William C, DLL, Univ of Houston, 3801 Cullen Blvd, Kouston, $\mathrm{Tx} 77004$

DTAAIO, Carlo, DLL, Dept FL, Iouisiana State Univ, Baton Rouge, Ia 70803

DIVIMCENZO, Prof Vito, DLL, Villanova Univ, Spring and Hilldale Rd, Malvern, $\mathrm{Pa} 19355$

DODGE, James W, Middlebury College, Sunderland Hall, Middlebury, vt 05753

DOHERTY, J C, Chmn Lang Dept, st Malachy S HS, Ieinster St, St John, N B, Canada

DOVIING, Charles, DLL, Baldwin Hallace College, Berea, Oh 44017

DOHNING, Mrs B, W Texas State Univ, Canyon, Tex 79015

DRAGONE, Dr 0, Dir MLS, AIC, state St, Springfield, Ma 01109

DRAKEFORD, Carolyn S, 2226 l/2B Hampton St, Columbia, s C 29204

DRUMMOND, Donald I, Audio Coord, 1001 Howard St, San Antonio, Tex 78212

DUCHARIE, Sister M J, Chmn ML, Rivier College, Nashua, N H 03060

DUKAS, Dr Vytas, FLL, German-Russian Dept, 5402 College Ave, San Diego, Ca 92115

DULKA, Richard, Dept FL, Eastern Illinois Univ, Charleston, Il 61920

DUNAWAY, Agnes, Chmn FL, Riverside HS, 1615 Locust St, Milwaukee, W1 53211

DURHAM, Bill, 201 Strode Tower, Clemson Univ, Clemson, s C 29631

DUVAL, F A, Chmn Class/Mod Lang, Cornell College, 710 th Ave $\mathbb{N}$, Mt Vernon, Ia 52314

$$
-\mathbf{E}--
$$

EBERHARDT, George M, Tech Dir IL, Drew University, BH 217, Madison, N J 07940 ECONOHOS, Prof R M, Pace College, 41 Park Row, New York, N Y 10038

EGGMAN, H, Chm Boys Div I, Bis Reilly Diocesan HS, F Lewis LI Expresstray, Fresh Meadows, N Y 11365

EILERTSON, E J, AP of German, Carroll College, 414 W Park Ave, Waukesha, Wi 53186

EISENSTADT, Ralph A, DIL, West Chester state College, FL Dept, West Chester, $\mathrm{Pa} 19380$

EISt:ERTH, Judy, 2323 Rowena Ave No 3, Ios Angeles, Ca 90000

EK, James D, DLL, Western Michigan Univ, Kalamazoo, Mich 49001

ELDON, Mrs Jane, DIL, N Central College, Naperville, Il 60540

EIDRIDGE, Richard H, P O Box 46, W V W C, Buckhannon, WV 26201

ELIFSON, Mrs Iucy, Waukesha HS, $400 \mathrm{~N}$ Grand Ave, Waukesha, Wi 53186

ELLIS, Prof Ray, Hardin-Simmons Univ, Abilene, ix 79601

EUENDORF, William E, DLU, Western Michigan State College, Bellingham, Wa 98225

ElARRY, Edmond L, Chmn FL, Milford Academy, 150 Gulf St, Milford, Ct 06460

ENILIENNE, Sister Therese, DIL, Annhurst College, Woodstock, Ct 06281

EPFER?, Dr Franz, Assoc Prof German, Univ of Hew Brunswick, Fredericton, N B, Canada

EPPINK, Alice J, Iib, Center for Applied Iing, 1717 Wass Ave NW, Washington, D C, 20036

ERICKSON, Mrs Evelyn, Box 104, Mahopar, NY 10541 


\section{Membership Directory}

ERIKSON, Prof Carl T, P O Box 449, Shorehem, NY 11786

ESPMNOSA, Lorenzo, IL Supv, Univ of California, Riverside, Ca 92502

EVING, B R J r, DLL, Washington and Iee Univ, Dupont 102, Lexington, Va 24450

$--F--$

FAIRLEY, Mamie, Head FL, Magnolia High School, Box 428, Moss Point, Ms 39563

FANTIVI, Alvino E, 205 West $33 r^{d}$ St, Austin Tx 78705

FARRELL, Br, CFX, Chmn ML, Ryken HS, Leonardtow, MA 20650

FAULKRER, James C, State Univ College, 17 Cedar Ridge Rd, New Paltz, NY 12561

FECTEAU, Leo J, Head, FL Dept, Barrington Public Schools, Barrington HS, Barrington, RI 02806

FEINBERG, Rosa Castro, Robert E lee Jr High, $3100 \mathrm{NW}$ 5th Ave, Miami, Fl 33127

FEISHER, Dr Villiam M, Head, Dept FL, Univ of Evansville, Bvansville, In 47704

FERAL, Dr Pierre, P O Box 877, Station B, Ottawa 4, ontario, Canada

FERGUSON, Iou, Chmn FL Dept, Glenbard East HS, $1014 \mathrm{~S}$ Main St, Lombard, II 60143

FICARRA, A V: Asst Prof, Vestern Conn State College, 181 White St, Danbury, Ct 06310

FIELD, Douglas A, Dept FL, Maui Conam College, Kahului Maui, Hi 96732

FINN, B J, DLL, Skill Center, 1485 Market St, San Francisco, Ca 94103

FISHER, Ronald D, DLL, LaSalle College, 20th St at Clnoy Ave, Fhiladelphia, $\mathrm{Pa} 19141$

FITCH, C Bruce, DLL, Transylvania College, $300 \mathrm{~N}$ Broadway, Lexington, Ky 40508

FLAMING, Donald N, 209 Baker St, Keene, NH 03431

FOBES, Dorothy, Youngstown State Univ, Youngstown, Oh 44503

FLUCKIGER, Prof P Fred, Dir, Abeeilung flir angewandte Iinguistik der Univ Berne, Länggasse 7, 3012 Berne, Switzerland

FOURHIER, Robert, Consit FL, St Dept of Educ, 17 Webster St, Suncook, NH 03275

FOWLER, Betty, Conslt FL, Dept Ed, Stanislaus City S C, P O Box 1697, Modesto, Ca 95354

FRANCISCO, David, Dir, Dial Access, Forest Park College, 5600 Oakland, St Louis, Mo 63110

FRANKS, Gene, Dept FL, N Texas State Univ, Denton, Tx 96203

FRECHETTE, Ernest A, ML Dept, Florida State Univ, Tallahassee, Fl 32302

FREEMAN, Robert, West Bend High School, 710 S Main St, West Bend, W1 53095

FREVAL, Daniel, Lab Dir, Dickinson College, Dept ML, Carlisle, Pa 17013

FRIEDMAN, Frank M, DLL, Flint Conm Jr College, 1401 E Court st, Flint Mi 48503

FRITSCH, Hans J, P O Box 61, Whately, Ma 01093

I'ROST, Arthur, DLL, Princeton Univ, 304 E Pyne Bldg, Princeton, N J 08540

$$
\text { --G-- }
$$

GABEL, Dorothy, Chmn, FL Dept, Resurrection HS, $7500 \mathrm{~W}$ Talcott, Chicago, Il 60631

GARFINKEL, Alan, Tech, Dept Ed Assoc, Oklahoma State Univ, Stillwater, rik 74074

GARTMGER, Ermal E, DDL, Blake Annex, Univ of Kansas, Lawrence, Ks 66044

GAUDINO, V A, O I S E, Nodern Lang Cen, Toronto 181, Canada

GAUTIER, Roger T, DLL, Univ of Saskatchewan, Saskatoon, Sask, Canada

GEMMATO, Geno R, Dir Audio-Labs, Tufts Univ, Medford, Ma 02155

GENDREAU, N, ML Dept, Rhode Island College, 600 Mt Pleasant Ave, Providence, R I 02909

GENDRON, Raymond, Head, Lang Dept, Seekonk Sr HS, Seekonk, Ma 02771

GENNAULA, Mrs M G, 5872 Kings School Rd, Bethel Park, Pa 15102

GIELLA, Miguel Angel, Spanish Dept, Carleton Univ, Colonel By Drive, Ottara, Ontario, Canada

GILL, S Rosenda, DML, LL Coord, Regis College, Weston, Ma 02193 


\section{Membership Directory}

GIONET, Arthur J, DLL, N Texas State Univ, Denton, Tx 76203

GLICKMAN, Maurice, 1047 S Sierra Bonita Ave, Los Angeles, Ca 90019

GLODELL, Marcus, Prof of Spanish, 251 Belmont St, Worcester, Ma 06105

GOLDSTEIN, E M, Prof Ed, Fac of Ed/Grad School, Univ of Ottawa, Ottawa 2, Ontario, Canada

GOLDSHORTHY, T R, DLL, Univ of Visconsin, 866 Van Hise Hall, 1220 finden Dr, Madison, Wi 53706

GONZALES, Angelita Floro, California State College, 27669 Loyola Ave, Hayward, $\mathrm{Ca} 94545$

GOODRICH, Albert A, DIL, Joint School Dist No 1, Three Lakes, Wi 54562

GORDON, Alan M, DLL, Dept of Ital and Hisp Studies, Univ of Toronto, Toronto, Ontario, Canada

GORDON, Ian B, P O BOx 507, Wingate, N C 281'74

GORE, Peter B, DL, Deerfield Academy, Deerfield, Ma 01342

GOULOT, Sr Noella, Notre Dame College, 2321 Elm St, Manchester, N $\mathrm{H}_{03104}$ GREGORY, Clara F, 1202 Newning, Austin, Tx 78704

GREIPP, Joseph F, I D I T, 119 Sherwood Dr, Greenwood Fk, Blackwood, N J 08012

GRITTER, Frank, St Supv/MFL, Wisconsin Dept Public Inst, 710 Woodward Dr, Madison, Wi 53704

GROSJEAN, Glen, DLL, Univ of California, B-40 Dwinelle Hall, Berkeley, Ca 94720

GROSS, Mrs Stephania E, Bowling Green State Univ, Univ Hall, Bowling Green, Oh 43402

GRUNDSTROM, Allan W, Asst Prof of French, Bucknell Univ, Lewisburg, Pa 17837

GUENETTE, Paul G, DLL, Town of Brookline, Town Hall, 333 Wash St, Brookline, Ma 02146

GUERTIN, Sister M George, Mt St Mary College, Hooksett, N Y 03106

GUEST, Dr James, Head, Lang Dept, Pensacola Jr College, 1000 College Blvd, Pensacola, FI 32504

GUGGER, Edward M, Head, Dept of FL, Medford HS, Medford, Ma 02155

GUITTON, Dr Jean, Chmn, MI, Georgia College, Milledgeville, Ga 31061

GUSTAFSON, Marjorie, DLU, MacMurray College, 1419 Lakelawn Dr, S Jacksonville, Il 62650

$$
--\mathrm{H}--
$$

HAGEN, Mrs Donald F, DLI, Fairleigh Dickinson Univ, 285 Madison Ave, Madison, N J 07940

HALL, Alvin L, Dept FL, Univ of SC, Columbia, SC 29208

HAIL, Wendell H, Asst Prof Spanish, Box 214 Univ Station, Provo, Utah 84601 HAMOMD, Dr, Sp Lang Pub Service, 211 S Main St, McAllen, Tx 78501

HAMSON, Erwin M, DLL, Univ of Michigan, 1401 Mason Hall, Ann Arbor, Mi 48104 HArSON, David A, APR, Ger and Slavic Lang, Brandeis Univ, Waltham Ma 02154

HANSON, J ames, Chmn, Dept of ML, Adrian College, Mahan Hall, Adrian, Mi 49221

HAPBURN, Eric, 25 Colvin ct, Old Bridge, N J 08857

HAREHOOD, Glenn, Humber College of $\mathrm{A} \mathrm{A}$ and $\mathrm{T}$, Kumber College Blvd, Texdale, Ontario, Canada

HARRISON, Br Edward, French/Spanish Instr, Walsh College, 2020 Easton Rd, Canton, oh 44720

HARRISON, C Norris, Dir A V Serv, Washington College, Chesterton, Md 21620

HARTLEY, Ernest B, DLL, Univ of SC, Columbia, SC 29208

HARTLEY, G. Christopher, Head, Lang Dept, The Peddie School, Hightstown, N J 08520

HAUGHTON, V, Chmn French Dept, MacDuffie School, Springfield, Ma 01107

HAUK, Gunther, Dept ML, Allegheny College, Meadville, Pa 16335

HAWKIIS, Paul Jr, Ger Chmn, WV Univ-Parkersburg Ctr. 110 16th St, Vienna, b V 26101

HAYASAKA, Paul M, Loma Iinda Academy, 10656 Anderson St, Loma Iinda, Ca 92354 HAYDEN, Richard L, ML, Univ of Texas, El Paso, Tx 79968 


\section{Membership Directory}

HEIDINGER, Dr Maurice $M$, Assoc Prof of Ger, Dept of Ft, East Mt College, Billizgs, Mt 59101

HENDRICKSON, Robert L, 2905 Madison Ave, Granite city, Il 62040

HENNEBERGER, Robert, DLI, $170 \mathrm{~A}$ S B, U of Florida, Gainesville, Fl 32601 HERNANDEZ, Adele B, Instr of Span, 96 Falmouth St, Portland, Me 04103 HERON, Robert F, FL Dept Head, Bellingham HS, Bellingham, Ma 02019

HERRERA, Lazaro M, DLL, The Westminster Schools, $1424 \mathrm{~W}$ Pace Ferry Rd NW, Atlanta, Ga 30327

HEUBIEIN, Linda, DLL, College of St Teresa, Winona, Mn 55987

HEWSON, Dr J, Mem liniv of Newfoundland, St John S NFL, Canada

HILTS, Mrs Margarete, Loma Linda Univ, Riverside, Ca 92505

HIRSCH, Mrs Bernice G, DLL, Samford Univ, 300 Lakeshore Dr, Birmingham, Al 35209

HOLLAND, Reuben V, DLJ, Univ of Tennessee, Chattanooga, Tn 37403

HOPKINS, A H Jr, Apt G, 24 Jefferson St, Manchester, Ct 06040

HORSTMAN, William, DLW, Univ of Wisconsin-Milwaukee, Milwaukee, Wi 53211

HOWEL, Raymond, 1403 3rd Ave N, Seattle, Ha 98177

HOWLING, Robert T, Chmn Eng Human, New Haven College, New Haven, Ct 06505

HUFFMAN, Prof Donald T, W Virginia Univ, 781 Meadowbrook Rd, Morgantown, W V 26505

HILL, Alex P Jr, A P Lang, DLL, Texas Tech College, P 0 Box 4235, Lubbock, Tx 79409

HIMPHREY, Harvey J Jr, DLL, Fordham Univ, Bronx, N Y 10458

HIURIEY, Walter, Dept Ger-Cl Riss, Sacramento State College, Sacramento, Ca 95819

HURTADO, Herman, Dir Comm Lab, F L A, Atlantic Univ, Boca Raton, F1 33432

$$
- \text { I-- }
$$

IANNUZZI, Prof D A, Chmn ML Dept, Niagara Univ, P 0 Box 164, Niegara, N Y 14109 INES, Sr Maria, DLL, St Joseph State College for Women, 245 clinton Ave, Brooklyn, N Y 11205

IOUP, Georgette L, 402 v 22nd st N 3 F, New York, N Y 10011

ISSACHAROFF, Prof Michael, Dept of Rom L, Washington Univ, St Louis, Mo 63130

$$
--\mathbf{J}--
$$

JACKSON, Gordon, DLI, Capitol Univ, 2199 E Main, Columbus, Oh 43209

JACKSON, James W, DLL, West Michigan Univ, Dept of ML, Kalamazoo, Mi 49001

JACQUES, Fernand, Instr, Lakewood High School, 13429 Emerson Ave, Iakewood, Oh 44107

JALITNG, Hans, LL Research Project, Univ Stock-Strandbacken, Bromma, Sweden JAMIESON, H W, 22 Thronlee Cr NW, Calgary 47, Alberta, Canada

JANES, Joseph J, Hinsdale HS South, 7300 Clarendon Hills, Clarendon Hills, II 60514

JARLETT, Francis G, DLI, Central Connecticut State College, New Britain, Ct 06050

JERNER, Carl, 2600 Mission Bell Rd, San Pablo, Ca 94806

JOCHMANS, Robert P, Chmn, ML Dept, Union College, Ifincoln, Nb 68506

JOHNSON, Barbara, FL Dept, Washington State Univ, Pullman, Wa 99163

JOHNSON, Edward, 1250 s Ardmore, Villa Park, In 60181

JoHnson, Jane P, Asst Prof Ger, Bakersfield College, 1801 Panorama Dr, Bakersfield, Ca 93305

JOFNSON, Robert, DLL, Claremont Men's College, Claremont, Ca 91711

JONES, James A, Pennsylvania State Univ, S-320 Eurrows Bldg, University Park, $\mathrm{Pa} 16802$

JONES, R J, College of Ed, Univ of Toronto 70, 371 Bloor st W, Toronto 5, Ontario Canada

JULEL, Denis N P, Dept Rom Lang, U of New Brunswick, Fredericton, NB, Canada 
JULII, Sr, Head, French Dept, Maryvale-Trinity College Prep School, Valley Rd, Brooklandville, Md 21022

$$
--\mathrm{K}--
$$

KABLER, Irene, Mills Godwin Jr HS, 14800 Darbydale Ave, Woodbridge, Va 22191 KADAR, Dr G, DL, Napa College, Dept of FL, Napa, Ca 94558

KAMINSKI, Edmins J, Assoc Prof ML, Marietta College, Marietta, on 45750 KAUB, Shirley J, P 0 Box 2189, $545 \mathrm{~V}$ Dayton St, Madison, Wi 53703

KAUFKE, Dr Pierre, Dept of FL, Univ of West Fl, Pensacola, F1 32504

KAWARABAYASHI, Yusuke, Univ of the Pacific, Ilf5 Bernice Ave, Stockton, Ca 95207

KGENAN, Miss P, 2682 17th Ave, San Francisco, Ca 94116

KRLIMNGSR, Mrs Cesi, Lab Dir, Wilson College, Chambersburg, Pa 17201

KFISO, Sister Beth, DLI, St Mary of Woods College, St Mary of Woods, In 47876

KNISO, Priscilla, DLL, Newton Jr College, Washington Park, Newtonville, $\mathrm{Ma} 02160$

KTMP, Innwood $\mathrm{E}, 150$ West St, Wilmington, Ma 01887

KGMPRECOS, Joseph, DLL, Rumson Fair Haven Reg High, Rumson, in J 07760

KBRNEAIY, William P, DIL, Lexington HS, 7 Hancock Ave, Iexington, Ma 02173

KGNMEDY, Daphene, Supv II, Dept MI, Freed-Hardenan College, Henderson, Tn 38240

KCNMEDY, Dora F, 4806 Harvard Rd, College Park, Md 20740

KGRR, Charlotte W, Supv IL, Occidental College, 2600 Campus Rá, Ios Angeles, Ca 90041

KIBART, Mrs Sylvia, FI Dept, E Texas State Univ, Commerce, Tx 75428

KHEKIL, R D, Asst Prof, Dept ML, Oregon State Univ, Corvallis, or 97331

KTMML, Dr Arthur S, FL Dept, Western Washington State College, Bellingham, Wa 98225

KINSEL, Anne, HS Libr, W Lafayette Comn College, Ieslie and Grant, Iafayette, In 47901

KNOYVTAR, Egetemi, POB 483, Budapest 5, Hungary

KONHIIR, George E, DL, St Procopius College, Hisle, Il 60532

KOENEN, Shirley, 2420 Appleside Blvd, Clarkston, W Va 99403

KOPP, Dr W LeMarr, Asst Dean, Pennsylvania State Univ, 138 Sparks Bldg, University Park, $\mathrm{Pa} 16802$

KOVARY, Prof Tom T, DLU, State Univ of NY, College at Cortland, Cortland, N Y 13045

KOZORIZ, Commandant G, Canadian Forces FIS, Dept of Nat Def, Ottawa 4, Ontario, Canada

KRUMM, Hans-JUrgen $\mathrm{krumm}, \mathrm{M} \mathrm{A}$, Zentrum flur neue Iernverfahren, Minzgasse 11 , D 74 Tubingen, $\%$ Germany

KRUSE, Bdward I Jr, Lect in Bd, Canisius College, Buffalo, N Y 14208

KRYIITZ, ROY I, DIf, FL Dept, NFew Mexico State Univ, University Park, N $\mathrm{M}, 88001$

KUIIEKA, Betty, Chmn, FL Dept, Kaimuki HS, 2705 Kaimuki Ave, Honolulu, Hi 96816

KURTYAMA, Shoichi Prof, Waseda University, 3-12-16 Shimoiguse Suginami, 167 Tokyo, Japan

$$
-\mathbf{L - -}
$$

IABELLE, Quentin, Windsor Mountain School, Lenox, Ma 01240

IACEFIELD, Arch S, Sr Instr, Col Iib Univ of Kentucky, Henderson Corm College, Henderson, Ky 42420

IAFAURY, P G, DLL, Univ of St Michael S College, $50 \mathrm{sr}$ Joseph Sr, French Dept, Toronto, 5, Ontario, Canada

LAGUEX, Raymond $J$, Act Chmn, Dept of Lang, Farmington State College, Farmington, Me 04938

IAILY, Dele V, DLL, Marquette Univ, Milwaukee, Wi 53233

IAM, Tian-Yee, Stanford Univ, P 0 Box 3266, Stanford, Ca 94305 


\section{Membership Directory}

IANDRY, Sister Monica, Notre Dame HS, 910 NI Easton Ave, Crowly, Ia 70526

LANGE, Dale I, Assoc Prof, Univ of Minnesota, 152-A Pelk Hall, Minneapolis, in 55455

IAPP, Donald, Prof Span, Aurora College, 347 Gladstone, Aurora, Il 60507

IARSON, Elinor C, Lab Dir, Scotch Plains-Fanwood kS, Westfield Rd, Scotch Plains, in J 07076

IATHROP, Prof Thomas A, Mt St Mary's College, Ios Angeles, Ca 90049

IAVELLE, Adrienna, DLL, Cumberland HS, Mendon Rd, Cumberland, RI, 02864

IAY, Amado M, Prof Span, Gustavus Adolphus College, Box 1413, st Peter, Mn 56082

IFAHY, S Peter, DHT, Wisconsin State Univ, Stevens Point, Wi 54481

IEDIFSKY, Raymond J Jr, DIL, New Trier HS East, Winnetka, Il 60093

IELIEVERE, I F, CA, Lower Canada College, 4090 Royal Ave, Montreal 261, $\mathrm{PQ}$, Canada

LABMYE, J C, DIL, MCGill Univ, Montreal, PC, Canada

LIMSING, Arvelia, DLI, Instr Spanish, St Ambrose College, Davenport, IA 52803

LBONE, Arthur A, 2593 Shelly Dr, Indiana, Pa 15701

IEvINSON, Mrs B, Supv Lab, Brooklyn College City Univ, Bedford Ave and Ave $\mathrm{H}$, Brooklyn, N Y 11210

LEWIS, David W P, Asst Prof of ML, Lakehead Univ, Thunder Bay, Ontario, Canada

LIJI I, Marcel, Iect, Rom IL, CCNY, 133rd and Convent Ave, N Y, N Y 10031

LTGHTCAP, David W, A V Dir, Susquehanna Univ, Selingsgrove, Pa 17870

ITNGBLAD, Dr Torsten, Lärarhögskolan I D, Gume, 41314 Göteborg, Sweden

IINDBERG, R H, Chmn Lang Dept, Gov Dunmer Academy, Bayfield, Ma 01922

ITIDNER, Iuis, DLL, Stephens College, Columbia, Mo 65201

LIDDQUIST, Mrs Pirkko Alex von Humboldt Kielil, Turun Ylipois to-Turku, Suomi, Finland

LITILE, Mrs IInda $W$, Dept of FL, Northern Illinois Univ, DeKalb, Il 60115

Io RE, Dr A G, DIL, Univ of N C, 105 Dry Hall, Chapel Hill, N C 27514

IOUBERT, Sister Elaine, College of St Scholastica, Duluth, Mn 55811

LOVERSO, Mrs Rosabianca, Dept French and Ital, Sacramento State College, Sacramento, Ca 95819

IOWENTHAL, Robert, 5700 Monroe St, Hollywood, FI 33023

IUIKART, Tim, DLL, Ashland College, Ashland, oh 44805

IJUMPRT, Rolf A, Univ of Dubuque, German Dept, Dubuque, Ia 52001

$$
--M-
$$

MACINKO, John, DFLL, old Main Bldg, U of Colorado, Boulder, Co 80300

MACINNIS, Sr Rita M CND, Xavier College Library. Box 760 , Sydney, N S, Canada MACKEY, Iola A, $4848 \mathrm{~N}$ Woodmere, Fairway No 7, Scottsdale, Ar 85251

MAGIIL, Dr Robert, DII, Towson State College, Dept ML, Baltimore, Md 21204

MALI, Pierce, Head, Dept ML, Princeton Day School, The Great Rd, Princeton, N J 08540

MANOIIS, John C, DLI, Mary Wash College of Univ of Virginia, P O Box 1126, College Sta, Fredericksburg, Va 22401

MANTINI, Mrs Barbara, N Hennepin State $\mathrm{Jr}$ College, 7411. 85th Ave N, Minneapolis, Mn 55428

MARESCHAL, Roger E, 45 Belle Meuniere Sud, Neufchatel, PQ, quebec, Canada

MARKLE, Dorothy, Asst Prof, Newark State College, Morris Ave, Union, N J 07083

MARSHALL, Prof D W, Vancouver City College, 912 W I2th Ave, Vancouver 9, B C, Canada

MARSHALL, William H, Asst Prof of Lang, Univ of Maine, Augusta, Me 04330

MARTI, Richard, 2369 Williams Apt C, Columbus, Oh 43202

MARTIN, Br James, CSC, Holy Cross HS, 26-20 Francis, Lewis Bl, Flushing, N Y 21358

MARTIN, Robert I, 2434 Shamrock Dr, Bowling Green, Ky 42101 


\section{Membership Directory}

MARTIN, Willard M, DLI, Pennsylvania State Univ, 6 sparks, Univ Park, $\mathrm{Pa} 16802$

MARTDEE, John, Chmn Span Dept, New Trier Twp HS West, 7 Happ Rd, Northfield, Il 60093

MARXHEIMER, Edward, DLL, Univ of Alberta, Edmonton, Alberta, Canada MARZOLF, Arnold H, North Dakota State Univ, ML Dept, Fargo, ND 58102 MASON, George D, 22 Industrial PK Rd, Hingham, Ma 02043

MAXWELL, Williem C, Head, Lang Dept, Tabor Academy, Box 372, Marion, Ma 02738

MCCLINTOCK, Geo I M Jr, 1825 McLaughlin Run Road, Pittsburgh, Pa 15241

MCCULIOUGH, J T, Dept Span and Port, Sacremento State College, Sacramento, Ca 95819

MCCUNE, Jerry, DII, 120 Ballantine Hall, Indiana Univ, Bloomington, In 47401 MCGETTRICK' Helen, 115 st stephen St, Boston, Ma 02115

MCKEAN, Thomes, HS Library, 301 McKennen's Church Rd, Wilmington, De 19808

MCMANYS, Rev J, Univ of St Thomas, 3812 Montrose Blvd, Houston, Tx 77006

MEADE, J ames J Jr, DLL, Brockton HS, 35 McDevitt Rd, Randolph, Ma 02368

MEESK, Mrs Carol, 1828 W Pensacola St, Apt B-14, Tallahassee, Fl 32304

MERCEDES, Sister Ima, Instr, Chestnut Hill College, Philadelphia, Pa 19118

MERCHANT, ROy D, P O BOx 382, Athens, on 45701

MERSHON, Homer, Dept of Ed, Edinboro St College, Edinboro, Pa 16412

MESS, Mary J ane, Chmn, FL Dept, Ottawa Twp HS, 211 E Main St, Ottawa, Il 61350

MESSNER, C A, Assoc Prof ML, Carleton College, Northfield, Mn 55057

METZGAR, Grace H, 1420 Montgomery, Univ of San Francisco, San Francisco, Ca 94133

MICKO, Micheel, DLL, FMC College, Chester, Pa 19013

MICOZZI, Arthur L, Supv FL, Board of Ed, Baltimore Co, 2401 Lampost Lane, Baltimore, Md 21234

MIKESELL, Norman L, Dir of Instr Equip Systems, Rm 113, CA Bldg, 925 W Michigan St, Indianapolis, In 46200

MItIJER, J B, Wittenberg Univ, Springfield, oh 45501

MILIER, Martha R, Admn Asst, Harvard Univ, ML Ctr, Boylston Hall G3, Harvard, Cambridge, Ma 02138

MILIER, Robert C, Lang Dept Chmn, Thatcher School, Ojai, Ca 93023

MILIER, Shelby C, 115 Dudley Rd, Cochituate, Ma 01778

MIIIS, Dr Dorothy hurst, Dept FL, Chapman College IL, Orange, Ca 92666

MITTAC, Marlin C, 710 Garfleld Ave, Aurora, Il 60506

MITTON, R C Laurie, DLL, Millersville state College, Millersville, Pa 17551

MOEN, Mary E, Instr, Anoka Ramsey State Jr College, 7154 NE Riverview Terr, Minneapolis, Non 55432

MONGIILO, Frances, 179 Huntinghill Ave, Middleton, Ct 96457

MOORE, Dr J Michael, Mesa College, San Diego, Ca 92111

MORAN, Sr M Catherine OP, Edgewood College Sacred Heart, 855 Woodrow St, Madison, Wi 53711

MOREAU, Val Guy, Chmn FI Dept, Portsmouth HS, Portsmouth, RI 02871

MORELY, Margaret C, DIL, Teachers College, Columbia Univ, Box 66, 525 w 120 th, NY, NY 10027

MORGAN, Mrs D K, Dept of Rom L, Univ of Alberta, Edmonton 7, Alberta, Canada

MORGAN, Margot, DLL, Sacred Heart Dominican College, 2401 E Holconbe, Houston, TX 77021

MORRISON, Joseph F, Audio Info Services, Univ of Illinois at Chicago Circle, Box 4348, Chicago, Il 60680

MORRISON, Robert R, C M L, Southern Missionary College, P O BOx 475, Collegedale, in 37315

MOSER, Worth S, Chm, Dept FL, Darlington School, Rome, Ga 30161

MOUNIAIN, Cliff, Dir FL, Nesa College, 1120 North Ave, Grand Junction, Co 81501

MLLFOLIAR, Charles V, Univ of R I, Ll Supv, Dept of Lang, Kingston, RI 02381 


\section{Membership Directory}

MURPHX, Harold T, Asst Prof Span, Marshall Univ, P O Box 238, Huntington, W V 25701

MUSSLER, Hans K, Dept of Lang and Phil, Utah State Univ, Logan, Ut 84321 MYERS, Thomas M, DIL, FLL, Colorado State Univ, Ft Collins, Co 80521

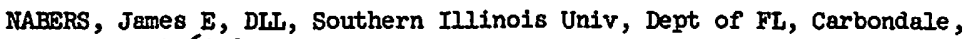
Il 62903

NASH, Donald S, Roberts Wesleyan College, North Chili, N Y 14514

NAVES, Carroll E, DLL, Barry College, Box 495, Miami, Fl 33161

NEARHOOF, Orrin, Iowa Dept of Pub Inst, 2745 Bennett Ave, Des Moines, Ia 50310

NEISON, Mary H, 1800 Clarke Dr, Dubuque, Ia 52001

NEMMI, Mrs Monique, Fren Dept, Glendon College, 2275 Bayview, Toronto 317, Ontario, Canada

NEWMAN, James L V, Simmons College, 300 The Fenway, Boston, Ma 02115

NEVMARK, Leonard, Chmn, Dept of Iing, Univ of California, La Jolla, Ca 92037

NICHOLSON, Chas E, CMFL, Kansas State Dept of Ed, $120 \mathrm{E}$ loth St, Topeka, Ks 66612

NoVAK, Witold, DII, A-V Ctr, Monmouth College, Monmouth, Il 61462

NUFFER, J Stanley, FLL, Fortland State Univ, P 0 Box 751, Portland, Or 97207

$--0--$

OLSEN, Irving S, Dir Instr Materials, Valparaiso Univ, MFL, Rm 147, Valparaiso In 46383

OTIS, Bruce, City Univ of N Y, 158 E 7th St, N Y, N Y 10009

OWENS, Mrs Ida Marie, FL Dept, Ramopo HS, George St, Franklin Lakes, N J 07417

$$
--\mathbf{P - -}
$$

PARIS, B E, Assoc Prof, Faculty of Ed, Sec Dept, Regina Cernpus, Regina, Sask, Canada

PAST, Dr, Ling, Univ of Texas at El Paso, P 0 Box 180, El Paso, Tx 79999

PATRON, Gloria A, DLL, Louisiana State Univ, Lake Front, Hew Orleans, Ia 70122

PAUL, Christa E, DLL, Pennsylvania College of Pharm and Sc1, 43rd and Kingsessing Av, Philadelphia, $\mathrm{Pa} 19147$

PEDERSON, Carl, 10262 Gregory st, Cypress, Ca 90630

PELOQUIN, Paul D, Chicopee Comp HS, Rolf Ave, Chicopee Falls; Ma 01020

FENTA, Richard M, Coord FL, 38 Normandy Rd, lexington, Ma 02173

FEREIRA, Anton, DLL, Box 1524, Connecticut College, New Iondon, Ct 06320

FEREZ, Mario P, LL, Brevard College, Brevard, N C 28712

FETERSON, Paul W, Dir Dept ML, Gannon College, Erie, Pa 16501

FETHERBRIDGE, D L, Assoc Prof, Univ of Lethbridge, Lethbridge, Alberta, Canada

PETRIE, Dr Milton I, Dir AV/TV Ctr, Univ of Iouisville, Louisville, Ky 40208 FETRIZZI, Dr Daniel J, Coord FL, Eisenhower College, Seneca Falls, if Y 13148 PETRU, Mircea, SC Asst, Kingsborough Comm College, Box 700, Brooklyn, IN Y 11235

PETTIT, John A, Head, ML Dept, Marietta College, Marietta, Oh 45750

FEYRAZAT, J E, DLL, Roanoke College, Salem, Va 24135

PHILLIPS, Ed, High St, Winthrop, Me $\mathrm{O}_{4} 364$

PHIIIPPON, Christian P, 2707 Stuart St, Berkeley, Ca 94705

PHYLLIS, Sr M, Notre Dame Prep, 815 Hampton Lane, Baltimore, Md 21204

PICCO, Mas Elsie V, Lab Supv, Bennington College, Bennington, Vt 05201

PIOLA, Miss Gladys, 194 Washington St, 3111, Hartford, Ct 06106 


\section{Membership Directory}

PIEASANTS, Jeanne Varney, Prof Emeritus of French, 20 Dewey Lane, Hampton Bays, N Y 11946

POND, Sr M Ielia, DLL, Univ of Dallas sta, Dallas, Tx 75061

POFE, W1Iliam A, Dept MU, Keuka College, Keuka Park, N Y 14478

PRATT, Judith, Chmin FL, Oswego HS, W First St, Oswego, N Y 13126

PROBST, Glen, Michigan State Univ, East Lansing, Mi 48824

$-\infty--$

QUERCIA, Michael P, Belmont Pub Schools, 644 Pleasiant st, Belmont, Ma 02178 QUEYJA, Michael T, DLU, Univ of Mississippi, University, Ms 38677

QUINN, T, Grad Stud, Ohio state Univ, 1384 Jones Tower, Columbus, Oh 43210

$$
-\mathbf{R - \infty}
$$

RADTKE, J, Head, ML, Merquette HS, $3401 \mathrm{~W}$ Wisconsin Ave, Milwaukee, Wi 53208 RADUNZ, Herman G, II Asst, Vancouver City College, I-3548 $\mathrm{W}$ th Ave, Vancouver $8, \mathrm{BC}$, Canada

RAFFERTY, Mrs Josephine, 890 B Gatehouse Dr, Decatur, Ga

RAICHLE, Prof Louis P, 3 shelterview Dr, Staten Island, N Y 10304

REESE, Winston J, DLL, Univ of Iowa, 125 Schaeffer Hall, Iowa City, Ia 52240

REID, Constance I, Elgenmann Ctr, Indiana Univ, Bloomington, In 47405

REID, Mrs Francis, Supv LL, Davidson College, Davidson, N C 28036

REINER, Col Howard, 37 Walnut Ave, Wheeling, w v 26003

REITINGER, Dr Gottfried, Universitat Innsbruck, Fischnalerstrase 4, 6020 Innsbruck, Austria

RHODE, Sr Elizabeth, Chmn Lang Dept, Alverno College, 3401 s 39 st, Milwaukee, Vi 53215

RIVA, Prof, Haag Hall, Rn 304, Univ of Missour1, Kansas City, Mo 64110

RIVERS, Prof Wilga $M$, Dept of French, Univ of Iilinois, Urbana, Il 61801

ROBBINS, Margarette, Box $667 \mathrm{kS}$, U Sta, Abilene, Tx 79601

RODEWALD, Mrs Janet D, DLJ, FL Dept, Cen Michigan Univ, Mt Pleasant, Mi 48858

ROLFE, Dr O H, Chmn Dept FL, Univ of Montana, Missoula, Mt 59801.

ROMERI, Mrs E, Chmin FL, Chadwick School, 26800 s Academy Dr, Palos verdes Penin, Ce 90274

ROSHGOLD, E, 22 Mishmeret st, Afeka, Tel-Aviv, Israel

ROSSDAIE. Irs Ada, 435 East Bldg, 239 Greene St, N Y, N Y 10003

ROY, Prof J, Dept French, Ra A35I, Arts Blds, Lauren, Remsey Lake/Tian Univ, Sudbury, Ontario, Canada

ROY, Dr R, Languages Dept, 1577 Wall St E, Hinnipeg 3, Manitoba, Canada

RUBIr, Kathleen Kay, Regis College, $W 50 t h$ Ave and Lowell Blvd, Denver, Co 80221

RUPPRECHT, Rev, Melvin, DH, St Vincent College, Latrobe, Pa 15650

RUSCH, Norma, DLL, Iakeland Union HS, Minocqua, Wi 54548

RYAN, Mrs Carole Ann, Asst Prof of French, Illinois College, Jacksonville, Il 62650

$$
\text { -S-- }
$$

SABEAN, Allan T, Dir AV Centre, St Mary's Univ, Halifax, N S, Canada SADBERRY, Dr Ionnie, Prof of Spanish, Prairie View A and $M$ College, Prairie View, Tx 77445

SAMPON, Victor, Chmn FL, DLL, Wayland Academy, Beaver Dem, Wi 53916

SANCHEZ, Mrs Mary, Supv FL, $P 0$ Box 4688, Clearwater, FI 33518

SANTOMO, Benigno F, span Dept, Richbourgh Jr HS, 197 Pine Dr, Crestviev, F1 32536

SAUNDERS, Helen V, 1622 Franklin Ave, Charleston, W V 25311

SAWYER, Jesse 0, DLI, Univ of California, Berkeley, Ca 94720

SCARTH, Margaret, Asst Prof in Span, Erindale College, Univ Toronto, Toronto 5, Ontario, Canada

SCHAEFFER, U M, Supv II, Connecticut College, Box 1524, Hew Iondon, Ct 06320 SCHAUBACHER, Anne Marie, Chmn French Dept, Shipley School, Bryn Mawr, Pa 19010 


\section{Membership Directory}

SCHSFSKY, Carl $\mathrm{W}$, Inst Med, Univ of Portland, $5000 \mathrm{~N}$ Willamette Blvd, Portland, or 97203

SCHEWE, Edward, 419 S Taylor Ave, Oak Park, In 60302

SCEMITZ, Victoria, Assoc Dir, Schoonover Lab, Ohio Northern Univ, Ada, Oh 45810

SCHOBEL, Mrs Dianne, 7801 Sagamore Dr, Cincinnati, on 45236

SCHOENEELD, Aurora A, FLL Supv, Univ of Southwestern Louisiana, Lafayette, La 70506

SCHOLIIS, Eberhard, DLL, Plattsburgh State Univ College, Plattsburgh, N Y 12901

SCHWANK, Dennis M, 4376 Decliaration Dr, Indianapolis, In 46227

SCHIENKER, L I, Mgr, Univ of Guelph, 230 Edinburgh Rd S, Guelph, Ontario, Canada

SEARCH, Russel H, Dir AV Materials, Bucknell Univ, Lewisburg, Pa 17837

SEIDEL, J, LL, Univ of Victoria, P O Box 1700 , Victoria, B C, Canada

SENECAL, Jean Paul, 289 Gardenville, Longueuil, P Q, Canada

SENG, Dr Mark W, 2505 Princeton Dr, Austin, Tx 78741

SEXTON, Mrs Eileen, Bronx Comm College, 120 E 184 St, Bronx, N Y 10468

SEYDOUX, Aline, Supv LL, 7101 w 80th St, Los Angeles, Ca 90045

SHAPIRO, Mrs Mary, St College, Bridgewater, Ma 02324

SHEARER, Miss Julie, 435-D Verbena Court, Orlando, Fl 32807

SHEREHAN, Prof Joseph H, Chmn Faculty Comm on LE, Georgetown Univ, Washington, D C 20007

SHErETS, Walter A, FL Supv, Bartholomew Cons Sch Drp, 1400 25th St, Columbus, In 47201

SHERBURNE, Robert, Cazenovia Central Sch, 2 Evergreen In, Cazenovia, N Y 13035

SHERRARD, David A, Chief AV Branch, DLIEC, US Naval Sta, Anacostia An, Weshington, D C 20390

SHERROW, Miss Renee, 315 E 70th St, Apt 2-A, New York, N Y 10021

SKIELDS, Marion D, MFL Dept Head, Dunedin HS, Dunedin, FI 33528

SHIREY, Mary M, 2419 Inngview Ave, SW, Roanoke, Va 24014

SIEVERS, Dale L, 333 Murfreesboro Rd, Nashville, in 37210

SIMMONS, Douglas J, Asst Prof, Dept of FL, Arizona State Univ, Tempe, Az 85281

SnfoN, Connie Lee, $303 \mathrm{~S}$ Alp, Bay City, Mi 48706

SIREVAAG, John, Head Ger Dept, Augustana College, Rocks Island, Il 61202

SKWAREK, Ruth, Instr, Muskegon City Comm College, 15088

SMITH, Alfred N, Asst Prof, Dept of Lang, Utah State Univ, Logan, Ut 84321

SMITH, Francis T, DLL, Seton Hall Univ, S Orange, N J 07079

SMITH, Dr Philip D, P C, West Chester State College, High and Rosedale Ave, W Chester, Pa 19380

SMITH, Flint, DLI, Purdue Univ, Dept of ML, Lafayette, In 47907

SMITHER, William J, Prof Span, Tulane Univ, DLL, New Orleans, La 70118

SNYDER, Elvin V, Prof of Span, E Mennonite College, Harrisonburg, Va 22801

SOKALSKI, Alex, P O Box 48, New Haven, Ct 06501

SONANDRES, William, Assoc Prof, Chmn FL Dept, Wilmington College, Box 1203, Wilmington, oh 45177

SOULE, Dennis, Instr, Bethany Lutheran Jr College, Mankato, Mn 56001

SPEAR, Frederick A, Assoc Prof of French, Skidmore College, Saratoga Springs, N Y 12866

SFETRINO, Anthony, Chmn FL Dept, Bassick HS, 1181 Fairfield Ave, Bridgeport, Ct 06605

SPINKS, O R, Dir SUNY at Albany, Humanities B-16, Albany, N Y 12203

STACK, Prof Edward, 3925 Arrow Dr, Raleigh, INC 27609

STANDISH, Mrs Marge E, 13 Post Rd, Pompton Plains, N J 07444

STEFFEN, Robert, Supv, Syracuse Univ Record Dept, 240 H S Crouse Bidg, Syracuse, N Y 13210

STEINBERG, Adrian L, Dir FLL, Temple Univ, 3rd Floor, College Hall, Philadelphia, Pa 19122

STEINEEYE, Geo, The Experiment in International Living, Putney, vt 05346 
STEHART, Harry E, 600 s College, Tursa, Ok 74104

SULLIVAN, James J, P O BOx 398, Jisle, 그 60532

SUITON, Louis M, Prof of French, Baylor Univ, Vaco, $\mathrm{Bx} 76703$

SWATI, Richard S, MLL Tech Dir, Marquette Univ, Milwaukee, Wi 53233

SWANSON, S A, DLU, Wisconsin State Univ, Oshkosh, W1 54901

SWIFT, Mirs Martha F, Morgan Park Academy, $2153 \mathrm{~W}$ 1llth St, Chicago, 그 60643

SYGODA, Ivan R, 22ll Graduate College, Princeton Univ, Princeton, N J 08540

$$
--\mathrm{T}--
$$

TANKI, Gifu Jyoshi, Daigaku Toorin Nagara, Gifu 500, Japan

TAYIOR, Welter C, Dept of Span, Upsala College, E Orange, N J 07019

TENNEY, ITs Beverly N, Contoocook Valley HS, Rte $202 \mathrm{~N}$, Peterborough, N H 03458

THERESE, Sr Marie, Chmn FL Dept, College of thite Plains, $52 \mathrm{~N}$ Broadway, White Plains, N Y 10603

THERRIEN, Marcella, DIL, 117 Margern Rd, Welland, Ontario, Canada

THEUMA, Jean R, Dir FL, Univ of Hawail, 2528 The Mall, Rm 303, Honolulu, Hi 96822

THIBAULT, Claude, Ecole Normale N-D-De Foy, Cap-Rouge, PQ, Canada THOMANN, Donaldo J, Chmn, ML, Pacific Union College, Angwin, CA 94503

THOMAS, Haley F, Inst, P O BOx 403, Univ of Richmond, Richmond, Va 23173

THORRE, E F, Asst Prof, Hest Liberty State College, West Liberty, W V 26074

THORNHIL, Donald E, Dir FL, Hestport Bd Ed, Westport, Ct 06330

TIBBETS, Jonathan, Acting DIL, Univ of Colifornia at Irvine, Irvine, Ca 92664

TOIRAC, Dorothy M, Chmn Lang Dept, Grace College, Winono Lake, In 46590

TOLMAr,, Jon M, Asst Prof, Indiana Univ, Dept Span and Port, Bloomington, In 47401

TOMKTI, Dr B, St Bonaventure Univ, St Bonaventure, N Y 14779

TORNATORE, Patricia N, Pemus Valley Area HS, Spring Mills, Pa 16875

TORRES, Sally, La Sierra Academy, 4900 Golden Ave, Riverside, Ca 92505

TORRIELII, A J, $820 \mathrm{~N}$ Michigan Ave, Chocago, II 60611

TOVEN, Audun, Asst Prof, Pacific Lutheran Univ, Tacoma, Wa 93447

TRACY, Robert $\mathrm{J}$, DLL, Ridgewood HS Ridgewood, N J

TRAHAN, Roger R, DLL, Assumption College, 500 Salisbury st, Worcester, $\mathrm{Ma} 01609$

TRIESCH, Dr Manfred, Dir Ger Ctr Boston, 170 Beacon St, Boston, Ma 02138

TRIPP, Mrs Gertrude M, DIJ, Elmhurst College, 190 Prospect Ave, Elmhurst, Il 60126

$$
--\mathrm{U}-\infty
$$

UMMEL, Carolyn M, Valley View HS, RR 2, Box 3370, Germantown, Oh 45327

$$
-\mathrm{V}-\boldsymbol{}
$$

VACANTI, Alfie A, 1506 Iverson st No 102, Oxon Hill, Md 20021

VANACORE, Sr Evangela, Op Dir, Ohio Dominican College, 1216 Sunbury Dr, Columbus, on 43219

VANDESTEWE, Mary D, Indiana Hill Jr-Sr HS, 7370 Shawnee Rd, Apt 3, Cincinnati, Oh 45243

VANG, Paul D, DLL, Thiel College, Greenville, Pa 16125

VANKERK, John $\mathrm{W}$, DLL, Indiana State Univ, $1532 \mathrm{l} / 2 \mathrm{~N} 7$ th st, Terre Haute, In 45705

VAN MERKENSTEIJN, E C, DLL, U of Pennsylvania, Philadelphia, Pa 19104

VAN TERESE, Glenn J, DLL, Sweet Briar College, P O Box 18, Sweet Briar, Va 24595

VAN WALK, Grace R, Grants Pass HS, 522 NE Olive, Grants Pass, Or 97526

VAN ZAANAN, Dirk B, Lab Dir, Rutgers State Univ, 175 Univ Ave, Conklin Hall, Newark, If J 07102

VAUPEL, Dean, College of Great Falls, Great Falls, Mt 59410 


\section{Membership Directory}

VELMEN, Prof Hugh E, Grand Valley State College, 615 Monroe Blvd, S Haven, Mi 49090

VESSEL, Bro Francis, St Michael S HS, Santa Fe, N M 87501

VICTOR, Michael, DLL, Jamestown Comm College, 525 Falconer St, Jamestown, N $Y 14701$

VIGIL, Neddy A, DLL, Univ of New Mexico, Dept of Mod and Class Lang, Albuquerque, N M 87106

VOLTRIC, Jon, LI, Ellis Hall, Ohio University, Athens, on 45701

$$
--\mathrm{Hil-}
$$

WADE, Harry A, Patrick Henry HS, 6702 Wandermere Dr, San Diego, Ca 92120

VALBRUCK, Harry, 313 Greenquist, Univ Bisconsin-Parkside, Kenosha, Wi 53140

WALSH, Sr Marie Andre IHM, St Edward S Univ, DLL, 3001 s Congress, Austin, Tx 73704

WARREN, Virgil A, 323 Corprew st, Fayette, Mo 65428

WATSON, Jack, DLL, The Karl E Weston Lang Ctr, Williams College, Williamstown, Ma 01267

WATSON, John A, Dir FLL, Virginia Union Univ, Richmond, Va 23220

WEBB, Dr Harry C, A-V Dir, College of St Thomas, st Paul, Mn 55101

WEBBER, Donald B, Asst Prof LL, The Univ of the South, Sewanee, In 37375

WEDKKAUF, Dr Arnold L, Asst Prof of Lang, Michigan Technological Univ, Houghton, Mi 49931

HHEETLEY, Dr V, Dir A/V Ed, 194 Boylston, Brookline, Ma 02146

UHITE, Richard J, DLL, Rich Central HS, Olympia Fields, Il 60461

WHITE, Sidney P, Dept Chmn, Morgan HS, Rt 81, Clinton, Ct 06413

WHITMORE, Allen P, Chmn Div of Lang and Lit, NE Missouri State College, Kirksville, Mo 63501

WIDAMIER, Karl, ML Instr, Atlantic Union College, S Lancaster, Ma 01561 WIESE, Peter, DLL, Southern Connecticut State College, 501 crescent St, New Haven, Ct 06515

WILKINS, $\mathrm{Dr}$, Dept Head FL, Iethbridge College Inst, Lethbridge, Alberta, Canada

WILKINS, Dr George W Jr, DLL, Tulane Univ, New Orleans, La 70118

WILIEY, Mr, Dir French Dept, Hyde School, Bath, Me 04530

WILLIAMS, Chas B, Lab Dir, State Univ College, Sheldon Hall, Oswego, N Y 13126

WILLIAMS, Raymond K, DLL, Farleigh Dickinson Univ, Rutherford, N Y 07070

WILLIS, Mrs Ione, RR 3, Milton, Ky 40045

WIN, Rev Prof Frank M, P O Box 24, Marlborough, N H 03455

WIPF, Joe, 121 E Knox Dr, W Lafayette, In 47906

WOHR, David L, 1831 N Broad St, Philadelphia, Pa 19122

WOODWORTH, Robert W, New Haven College, W Haven, ct 06516

WORTHINGTON, Mrs M L, AHDFL, Virginia State College, Box 428, Petersburg, $\mathrm{Va} 23803$

WNNN, Miss Carolyn, C-2 Georgetown Village, Spartanburg, SC 29301

$$
--Y--
$$

YALDEN, Mrs J M, Lect, Carleton Univ, Dept Span, Ottawa 1, Ontario, Canada YEAKEY, M L, Dept Chmn, Holland Hall School, 2640 S Birmingham, Tulsa, Ok 74214

YODER, Robert, Goshen College, 1005 South 8th St, Goshen, In 46526

YOSHISHIGE, George S, AV Ctr, Ieeward Comm College, 860 Fourth St, Pearl City, Hi 96782

YOUNG, Wm R, 3019 Monticello, Dallas, $7 \times 75205$

YZENBAARD, James, DLL, Univ of Kentucky, School of Letters and Lang, Lexington, Ky 40506 


\section{Membership Directory}

\section{$--\mathrm{Z}--$}

ZARR, Valois A, Chmn Ger Dept, East HS, 1134 S 17th E, Salt Lake City, Ut 94108

ZGARKA, M, 2nd Lang, Education Bldg, Rn 247, 3700 McTavish, Montreal 112, Canada

ZIERER, Prof Ernesto, Chmn Dept Ed, Idiomas Y Ling, Apt 315, Univ NAC, TruJ1.110, Feru

ZIMMERMAN, Sr Dorothy, DLL, Mount Mary College, Milwaukee, Wi 53222

ZIMERMAN, Ellen, DLL, Thornridge HS, 150-00 Cottage Grove, Dolton, Il 60419

ZMMERMAN, Mrs Margaret K, Chmn FL, Euclid Sr HS, 711 East 222nd st, Euclid, Ohio 44123

ZIRKO, Stephen, 1312 Crawford, Billings, Mt, 59102

\section{NATIONAL ASSOCIATION OF LANGUAGE LABORATORY DIRECTORS NALLD JOURNAL}

Name

Title

Institution

Address to which NALLD JOURNAL is to be sent:

Address

City ........... State ..... Zip.....

My school is paying the dues for NALLD Membership. Send the invoice to:
MEMBERSHIP

$\square$ New Member

U.S.A. (50 states)

Canada

Foreign Air Mail

Student (non-voting)

Library (non-voting)

APPLICATION

Return this form to:

Mr. Dale Lally

Treasurer, NALLD

Language Laboratory

Marquette University

Milwaukee, Wisconsin $\mathbf{5 3 2 3 3}$

Commercial organizations are invited to contact NALLD regarding associate membership.

Please mention NALLD Journal when writing advertisers 